Y. Papanikolaou • H. Palmer • M. A. Binns •

D. J. A. Jenkins · C. E. Greenwood

\title{
Better cognitive performance following a low-glycaemic-index compared with a high-glycaemic-index carbohydrate meal in adults with type 2 diabetes
}

Received: 22 July 2005 / Accepted: 23 December 2005 / Published online: 1 March 2006

C) Springer-Verlag 2006

\begin{abstract}
Aims/hypothesis: Transient hyperglycaemia, consistent with that observed with normal meal ingestion, may be detrimental to cognitive performance in adults with type 2 diabetes. This study determined whether minimising the postprandial increase in blood glucose through the ingestion of low- rather than high-glycaemicindex (GI) carbohydrate meals differentially affected cognitive performance in the postprandial period. Subjects and methods: Using a within-individual design, 21 free-living subjects (65 \pm 7.29 years) with type 2 diabetes consumed $50 \mathrm{~g}$ carbohydrate as a meal with either a low GI (pasta) or a high GI (white bread), or water on three separate mornings following an overnight fast. Neuropsychological tests were administered and plasma glucose concentrations measured. Results: Higher postprandial blood glucose AUC (gAUC) was associated with
\end{abstract}

Y. Papanikolaou · D. J. A. Jenkins · C. E. Greenwood $(\bowtie)$ Department of Nutritional Sciences, Faculty of Medicine,

University of Toronto,

150 College St, 3rd Floor,

Toronto, ON, Canada M5S 3E2

e-mail: carol.greenwood@utoronto.ca

Tel.: +1-416-9784261

Fax: +1-416-9785882

Y. Papanikolaou · C. E. Greenwood

Kunin-Lunenfeld Applied Research Unit,

Baycrest,

Toronto, ON, Canada

C. E. Greenwood

Department of Food and Nutrition Services,

Baycrest,

Toronto, ON, Canada

H. Palmer · M. A. Binns

Rotman Research Institute,

Baycrest,

Toronto, ON, Canada

D. J. A. Jenkins

Clinical Nutrition and Risk Modification Centre,

Division of Endocrinology and Metabolism,

St Michael's Hospital,

Toronto, ON, Canada poorer verbal memory (paragraph recall, $p=0.01$; word list recall, $p=0.012$ ). Both the GI of the carbohydrate meal and individual differences in response to meal ingestion contributed to the variation in gAUC and consequent memory recall. Bread consumption, relative to pasta, resulted in both a higher gAUC $(p<0.05)$ and worse delayed verbal memory performance (paragraph recall, $p=0.042$; wordlist recall, $p=0.035$ ). Additionally, performance following bread consumption was poorer than that following pasta on measures of working memory, executive function and auditory selective attention, while sustained attention showed no sensitivity to type of carbohydrate food consumed. Conclusions/ interpretation: Consuming $50 \mathrm{~g}$ of a low-GI carbohydrate meal, relative to a high-GI carbohydrate meal, generally results in better cognitive performance in the postprandial period in adults with type 2 diabetes, particularly in those individuals who experience the greatest food-induced elevations in blood glucose levels.

Keywords Carbohydrate - Cognitive performance . Glucose - Glycaemic index · Hyperglycaemia - Memory · Type 2 diabetes

Abbreviations BDI: beck depression inventory . gAUC: area under the blood glucose curve .

GI: glycaemic index $\cdot$ VPA: verbal paired associates .

WMS: Wechsler memory scale

Cross-sectional and prospective studies point to increased risk of cognitive decline in adults with type 2 diabetes $[1,2]$, especially in older adults demonstrating poor glycaemic control [1]. Declarative memory is especially vulnerable $[1,2]$, implicating functional disturbances in the hippocampus and related structures. Additionally, undesirable and transient excursions in blood glucose and/or insulin levels, secondary to insulin therapy-induced hypoglycaemia, and experimentally produced hyperglycaemia decrease cognitive function [3]. Specifically, acute hyper- 
glycaemic episodes in adults with type 2 diabetes, associated with either ingestion of $50 \mathrm{~g}$ of carbohydrate as a high-glycaemic-index (GI) food [4] or implementation of a hyperinsulinaemic/hyperglycaemic clamp [5], result in transitory cognitive dysfunction. Even normal fluctuations in the degree of hyperglycaemia experienced throughout the day in adults with type 2 diabetes appears to have a negative impact on cognitive tasks [6]. Thus, glycaemic variations typically experienced in individuals with type 2 diabetes, especially during the postprandial period, appear to be of sufficient magnitude to cause deterioration in cognitive function. Thus, the purpose of this study was to examine the impact of consuming high- and low-GI wheatbased carbohydrate meals, which produce markedly different postprandial blood glucose profiles, compared with a non-caloric control (water), on measures of cognitive function in adults with type 2 diabetes.

\section{Subjects and methods}

\section{Participants}

Twenty-one adults with type 2 diabetes (10 males, 11 females) who had not previously undergone cognitive testing were recruited. Fifteen subjects were treated with oral hypoglycaemic agents (metformin, $n=13$; sulphonylureas, $n=2$ ), while six were controlled with diet alone. Participants were included if their BMI and $\mathrm{HbA}_{1 \mathrm{c}}$ were below $40 \mathrm{~kg} / \mathrm{m}^{2}$ and $8.5 \%$, respectively. Exclusion criteria were insulin therapy, diabetic complications and substance abuse. The Beck Depression Inventory (BDI) [7] and the Shipley Institute of Living Scale (Shipley) [8] were administered at the first session to describe subjects on the basis of depression and general intelligence, respectively, but were not used as exclusion criteria. Informed consent was obtained from all participants and procedures were compliant with the Declaration of Helsinki and approved by the University of Toronto and St Michael's Hospital ethics committees.

\section{Procedures}

A within-subject design was used such that each participant acted as his or her own control. Following an overnight fast $(8-10 \mathrm{~h})$, subjects reported to the testing site medicationfree, for three randomly assigned sessions separated by a minimum of 1 week to minimise carryover effects. Subjects brought their medications to the site and took them immediately prior to meal treatments, but refrained from taking their medications until after the testing session was completed on the day that water was consumed. Subjects provided a fasting blood sample and then consumed, over a 10-min period, a low- or high-GI meal or water on each day. Cognitive testing began 15-min following each treatment.

The cognitive battery was identical on all three testing days, except that alternative test versions, randomly allocated across test days for each subject, were used. After consuming the breakfast or water meals, subjects first received three verbal memory tests in rapid succession, and measures of immediate recall were obtained for each test. Delayed memory recall (i.e. memory function following a determined passing of time, e.g. $30 \mathrm{~min}$ ) was assessed twice, each with a 30-min delay imposed, such that delayed testing occurred approximately $62 \mathrm{~min}$ (delay 1) and 100 min (delay 2) after starting the meal. A variety of nonverbal tests were administered during each delay period to measure attention and general executive brain functions.

\section{Blood glucose collection}

Five blood glucose samples were collected from subjects at each visit, using a finger-prick technique, to coincide with fasting ( $-5 \mathrm{~min}$ ), the beginning of cognitive testing (15 $\mathrm{min}$ after initiation of food ingestion), the beginning of each delayed recall period (62 and $100 \mathrm{~min}$ ) and on completion of testing (138 min). Blood glucose was assessed using the One Touch Ultra glucometer (LifeScan Canada, Burnaby, $\mathrm{BC}$, Canada). Blood glucose values obtained during the protocol were used to calculate the positive incremental area under the blood glucose curve (gAUC) [9].

\section{Dietary treatments}

The three dietary treatments were: (1) $250 \mathrm{ml}$ of water (control); (2) $50 \mathrm{~g}$ of available carbohydrate from a low-GI food: pasta (President's Choice, Toronto, ON, Canada) plus cheese (Kraft Light Mozzarella, Toronto, ON, Canada) and tomato sauce (Healthy Choice, Toronto, ON, Canada); and (3) $50 \mathrm{~g}$ of available carbohydrate from a high-GI food: toasted white bread (WonderBread, Toronto, Ontario, Canada) plus cheese and tomato sauce. The pasta was boiled for 6 min and the amount used was based on the package nutrition label; $55.8 \mathrm{~g}$ of pasta provided $42.5 \mathrm{~g}$ of available carbohydrate. Similarly, nutrition information on the bread packaging determined the amount of bread provided; $98.6 \mathrm{~g}$ of bread gave $42.5 \mathrm{~g}$ of available carbohydrate. In both dietary treatments, $37.5 \mathrm{~g}$ of cheese and $123.8 \mathrm{~g}$ of tomato sauce, added to improve palatability, provided the additional 1.5 and $6.0 \mathrm{~g}$ of available carbohydrate, respectively, giving $50 \mathrm{~g}$ of available carbohydrate for each treatment.

\section{Cognitive tests}

\section{Verbal memory recall}

Three separate standardised tests assessed verbal memory recall according to standardised procedures. All tests were pre-recorded in a professional sound studio and subjects heard information on audiocassette, thus standardising testing across subjects. Delayed memory was measured twice, 62 and $100 \mathrm{~min}$ after ingestion (Fig. 1, left inset). All 
test sessions were audio-recorded to allow verification of test scores.

The Hopkins Verbal Learning Test—Revised [10] was the first test administered to measure memory, which consisted of a list of 12 words (nouns). The word list was presented three times in rapid succession and subjects recalled the words immediately after each administration (IMM-1, -2, and -3). Subjects then recalled the word list, without an additional presentation of the word list, after a delay of $30 \mathrm{~min}$ (DEL 1) and $60 \mathrm{~min}$ (DEL 2).

Subsequent to the word list, standardised paragraphs (i.e. story) taken from the Wechsler Memory Scale (WMS)-R [11] and WMS-III [12] were administered. Test instructions according to the last revision [12] were used, such that two paragraphs were provided, the second paragraph being repeated twice to provide a second measure of immediate and delayed memory performance. An additional three paragraphs (taken from [13]) were required to have six different versions (i.e. two at each test session). Memory was assessed immediately after participants heard the paragraphs and after the two delay periods (30- and 60-min delays). Following the paragraph recall, the Verbal Paired Associates (VPA) subtest of the WMS-R [11] and WMS-III [12] was administered. Immediate and delayed recall were assessed following the format used for the word list and paragraph, with the exception that immediate recall was defined as the number of trials required to complete the task successfully. While all three versions of the VPA were taken from the WMS, one of the three versions was substantially more difficult relative to the other two. Consequently, data were adjusted for test version in the analyses to eliminate this potential confounder.

\section{Cognitive tests given during delay periods}

Alternate versions of the Digit Span, Trail-Making and Test of Everyday Attention were administered, in the same order, and repeated during each of the two delay periods.

The Digit Span Forward [12] is a test using digits that provides a measure of working memory (i.e. memory that temporarily holds information in the mind for current use).

Left- and right-handed versions, as well as their mirror images, of the standard Trail-Making A and B-Adult Form [14] were used to provide a measure of visual search, attention, motor function and general brain function.

The elevator task from the Test of Everyday Attention [15] measured attention. This task contained two parts-a

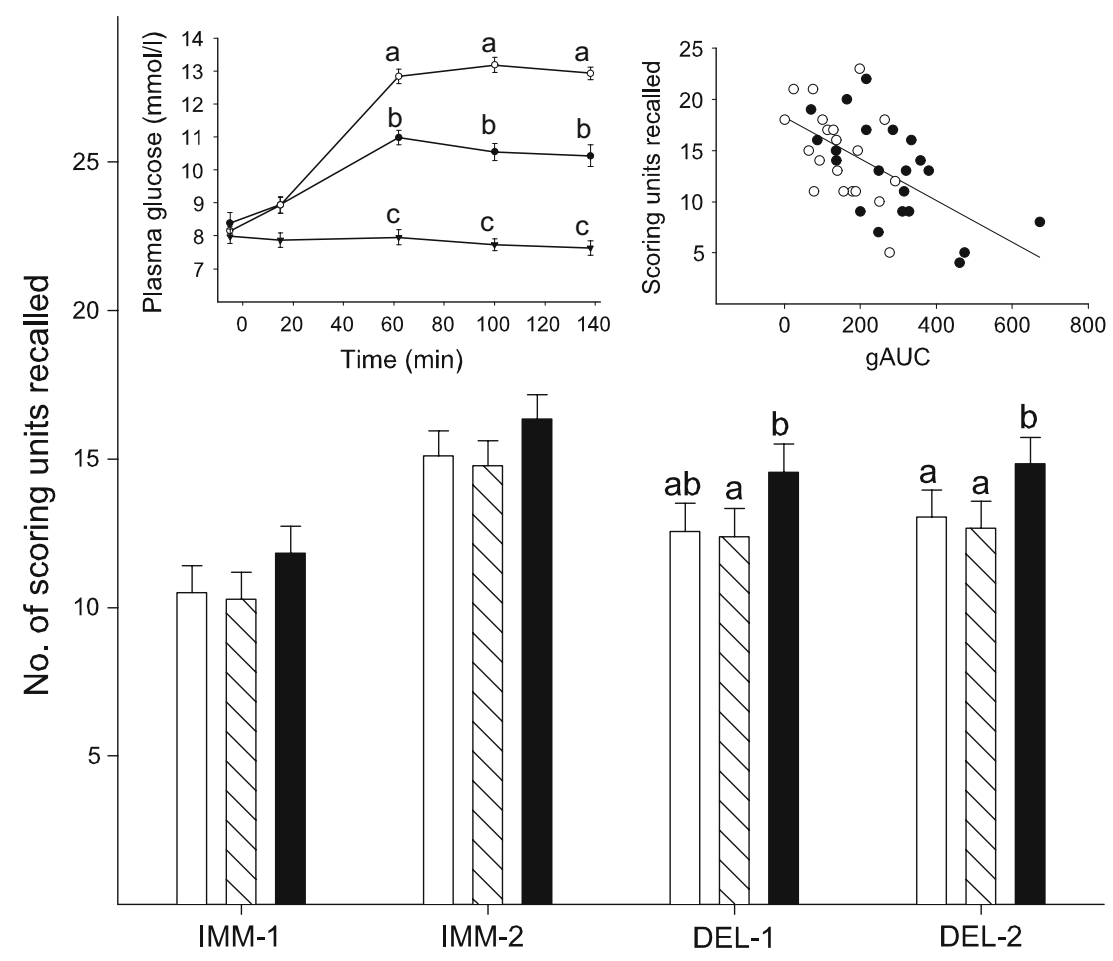

Fig. 1 Performance on paragraph recall. Mean $( \pm$ SEM) scores on the immediate (IMM-1 and -2) and delayed (DEL-1 and -2, second story) paragraph recall $(n=21)$ following consumption of water (white bars), white bread (hatched bars) or pasta (black bars). For each condition, means not sharing a common letter are significantly different $(p<0.05)$. Inset, left: mean $( \pm$ SEM $)$ plasma glucose concentrations in response to water (black triangles), bread (white circles) and pasta (black circles) consumption for all subjects $(n=21)$. For each time point, means not having a common letter are significantly different $(p<0.05)$. Thus, no differences between groups were observed at 0 or $15 \mathrm{~min}$ after consumption, but all three groups were significantly different at 60, 100 and $140 \mathrm{~min}$. The incremental glucose AUC was greater $(p<0.05)$ following bread consumption $(267 \pm 16)$ relative to pasta consumption $(147 \pm 10)$. Cognitive testing began $15 \mathrm{~min}$ following initiation of test meal consumption (time $=0$ ), verbal delayed recall being assessed following a $30 \mathrm{~min}$ (delay 1; time $=62 \mathrm{~min}$ ) and $60 \mathrm{~min}$ (delay 2; time $=100 \mathrm{~min}$ ) delay. Inset, right: delayed memory recall (delay 1 , second story) in relation to incremental gAUC for bread (black circles) and pasta (white circles). An inverse association is apparent, such that lower gAUCs following pasta and white bread consumption were related to better memory performance $\left(R^{2}=0.34, p=0.01\right)$ 
non-distraction component to measure sustained attention (i.e. focus for long periods of time) and a distraction phase to assess auditory selective attention (i.e. filter out distractions).

\section{Statistical analyses}

To assess the relationship between baseline cognitive performance and various health measures, multiple regression analyses were conducted (SAS-8.12; SAS Institute, Cary, NC, USA) using performance after water consumption as the response variable and all measured blood values, age, BMI, BDI and Shipley as predictor variables. Factors significantly associated with performance under the water condition (intelligence [Shipley], $\mathrm{HbA}_{1 \mathrm{c}}$ levels and depression [BDI]) as well as day of testing (to adjust for potential learning across the three test days) were used as covariates where appropriate in subsequent analyses.

A repeated measures ANOVA, at both the immediate and the delayed recall, examined the impact of each dietary treatment on test performance for each cognitive measure obtained. Contrast analyses were used to assess differences between treatments and linear regression analyses were used to examine the relationship between postprandial gAUC and cognitive performance. We calculated that sample size needed to be 21 individuals based on an alpha of 0.05 and a power of 0.80 . Statistical significance for all analyses was set at $p=0.05$.

\section{Results}

Study population

All subjects completed the study and were included in the analyses. There were no significant gender differences in subject characteristics (Table 1) or measures of cognitive performance. Based on BDI and Shipley performance, respectively, participants were not clinically depressed and demonstrated normal intelligence.

Blood glucose responses following dietary treatments

The gAUC was greater ( $p=0.035)$ following bread $(267 \pm$ 16) than after pasta consumption $(147 \pm 10)$. All dietary treatments differed from each other $(p<0.05)$ at 62,100 and

Table 1 Baseline subject characteristics $(n=21)$

\begin{tabular}{lc}
\hline Characteristics & Mean \pm SD \\
\hline Age (years) & $65 \pm 7.29$ \\
BMI $\left(\mathrm{kg} / \mathrm{m}^{2}\right)$ & $29.30 \pm 4.95$ \\
Beck Depression Inventory & $4.64 \pm 3.24$ \\
$\mathrm{HbA}_{1 \mathrm{c}}(\%)$ & $7.0 \pm 1.0$ \\
Fasting plasma glucose (mmol/l) & $8.20 \pm 2.50$ \\
Shipley Institute of Living Scale & $54 \pm 12.02$ \\
\hline
\end{tabular}

138 min, whereas no differences were seen at baseline and 15 min after meal ingestion (Fig. 1, left inset).

Dietary treatment effects on cognitive performance

\section{Word list}

A main effect of meal consumption on word list performance $(p=0.005)$ was observed, such that performance following the pasta meal was better than that observed following the consumption of either bread $(p=0.002)$ or water $(p=0.006)$, which did not differ from one another $(p=0.632)$. This benefit of pasta consumption was reflected in a greater rate of learning words over the first three immediate recalls (food $\times$ recall, $p=0.031$ ). Nevertheless, the absolute number of words recalled on the third immediate recall did not differ depending upon the food consumed $(p=0.226)$. By contrast, the dominant effect of meal consumption on delayed recall was observed with bread consumption. While no effect of food consumption was observed at the first delay $(p=0.508)$, performance following bread consumption was poorer than that observed following either pasta $(p=0.035)$ or water $(p=0.047)$ intakes, which did not differ from one another ( $p=0.224$; Fig. 2).

Regression analysis of the word list and gAUC revealed no significant associations for immediate recall. However, significance was nearly attained during delay $1(p=0.096)$ and a significant negative association was seen at delay 2 $\left(r^{2}=0.16, p=0.012\right.$; Fig. 2 , inset $)$.

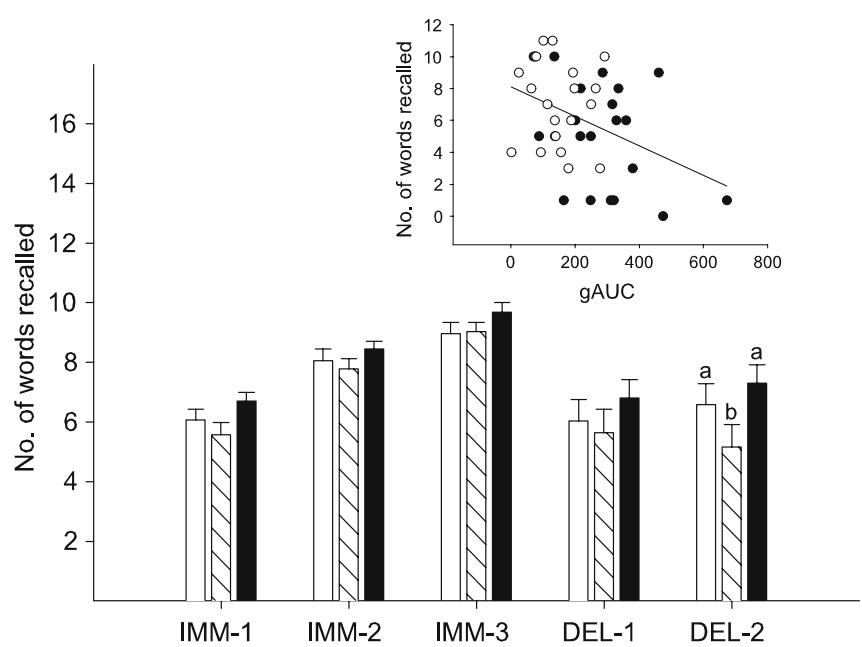

Fig. 2 Mean $( \pm$ SEM) performance on word list recall. Mean ( \pm SEM) scores on the immediate (IMM-1, -2 and -3$)$ and delayed (DEL-1 and -2) conditions of the word list recall task $(n=21)$ following consumption of water (white bars), white bread (hatched bars) and pasta (black bars). For each condition, means not sharing a common letter are significantly different $(p<0.05)$. Inset: an inverse association exists between gAUC and memory performance for the word list (delay 2), such that lower gAUCs following pasta (white circles) and white bread (black circles) consumption were related to better recall performance $\left(r^{2}=0.16, p=0.012\right)$ 


\section{Paragraph}

Like word list recall, paragraph recall performance showed a main effect of treatment $(p=0.005)$, such that performance following consumption of pasta was superior to that following bread $(p=0.001)$ or water $(p=0.036)$, which did not differ from one another $(p=0.197)$. Secondary analyses were subsequently used to separate immediate from delayed verbal recall performance. Meal effects were confined to measures of delayed recall on the second paragraph, with performance on pasta days being consistently superior to that observed following bread intake at both the first $(p=0.016)$ and the second $(p=0.042)$ delay period. Delayed recall after pasta consumption, relative to water, trended towards significance at the first delay $(p=0.055)$ and was superior to that following water intake at the second delay $(p=0.047)$. Bread did not differ from water at either the first $(p=0.592)$ or the second $(p=0.958)$ delay (Fig. 1).

gAUC was strongly associated with paragraph recall after the 30-min delay (delay 1), such that lower gAUCs following pasta and bread consumption were related to better memory performance $\left(R^{2}=0.34, p=0.01\right.$; Fig. 1 , right inset). At delay 2, a non-significant relationship was present, which trended in the same direction $(p=0.070)$.

\section{Verbal paired associates}

Unlike word and paragraph recall, a main effect of food was not apparent on the number of trials required for the VPA $(p=0.128)$. Nevertheless, there was an overall effect on the number of correct responses $(p=0.002)$, such that performance following water consumption was better than that following either pasta $(p=0.013)$ or bread $(p=0.001)$, which did not differ from one another $(p=0.465)$. Poorer task performance was consistently observed with bread and pasta consumption, relative to water, at both delay periods (immediate [number of trials required], water, 5.18 \pm 1.64 ; bread, 5.38 \pm 1.20 ; pasta, 5.20 \pm 1.37 ; delay 1 [number of correct responses], water, 6.43 \pm 1.77 ; bread, 5.39 \pm 1.45 ; pasta $5.50 \pm 1.51$; delay 2 [number of correct responses], water, 6.50 \pm 1.98 ; bread, 5.48 \pm 1.69 ; pasta, 5.63 \pm 1.74 ; all $p$ values $<0.02$ mean $\pm \mathrm{SD})$.

\section{Digit span forward}

A main effect of food was not apparent with Digit Span Forward performance, although it trended towards significance $(p=0.058)$. Given this trend, data were explored further, examining performance separately at each of the two delay periods (i.e. subjects performed the Digit Span Forward task twice on each test day, once during each of the two delay periods). Treatment effects were confined to delay 1 , such that performance was better on days when pasta was consumed, compared with performance on days when either water $(p=0.034)$ or bread $(p=0.045)$ was consumed, which did not differ from one another $(p=0.904)$. Again, gAUC following bread or pasta consumption was negatively associated with performance $\left(r^{2}=0.12, p=0.027\right)$. Performance did not differ between the water and bread conditions (performance during delay 1 , water, $10.13 \pm 2.03$; bread, $10.11 \pm 1.94$; pasta, $11.09 \pm 2.41$; mean \pm SD).

\section{Trail-making test}

No main effect of dietary treatments or delay period (task administration during the first vs second delay periods) was observed with time to complete Part A of the Trail-Making Test. Overall, better performance (reduced time) was observed on the second administration of Part B relative to the first $(p<0.001)$, but this effect interacted with treatment $(p=0.031)$. There was a greater improvement on days when pasta was consumed relative to bread $(p=0.006)$ and a non-significant trend for better performance on pasta days relative to water days $(p=0.080)$. The change in performance between the first and second administrations of the task did not differ between bread and water $(p=0.226)$. Similar statistical outcomes were observed when scores on Part A of the test were used as a covariate, or the data were expressed as a difference between Parts B and $\mathrm{A}$ (mean time reductions $\pm \mathrm{SD}$ were as follows: water, $18.19 \pm 2.32 \mathrm{~s}$, white bread $8.00 \pm 1.74 \mathrm{~s}$, pasta $21.67 \pm$ $2.89 \mathrm{~s}$ ). Thus, GI may not affect visual spatial function, but may be involved in higher brain functions dictated by the frontal lobes, at least as it relates to sustaining performance over time.

\section{Test of everyday attention}

Elevator task-no distraction When examining the elevator task without distraction, there was a significant treatment $\times$ delay period interaction $(p=0.028)$, such that the impact of food consumption differed when this task was performed during the first or second delay period. During the first delay period, better measures of sustained attention were apparent following bread $(p=0.044)$ and pasta $(p=0.003)$ consumption relative to water, but did not differ from one another (mean \pm SD: water, 6.19 \pm 1.33 ; bread, $6.52 \pm 1.40$; pasta, $6.76 \pm 0.77 ; p=0.229$ ). This benefit was not sustained throughout the testing period, with no differences between treatments being observed during the second delay $(p=0.343)$.

Elevator task-distraction A treatment $\times$ delay period interaction $(p=0.035)$ continued to be apparent when examining elevator task performance with distraction during each of the two delay periods. By contrast to the sustained attention, treatment effects on auditory selective attention were apparent in the second $(p=0.046)$, but not the first $(p=0.693)$, delay period. On days pasta was consumed there was better performance relative to water $(p=0.020)$ but not bread $(p=0.661)$. There was a trend for better performance following bread consumption compared with 
water $(p=0.054)$, which did not attain statistical significance (mean $\pm \mathrm{SD}$ : water, $8.14 \pm 2.61$; bread, $9.00 \pm 1.79$;

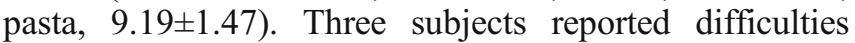
comprehending task instructions; specifically, they were not able to distinguish between the high- and low-pitched tones. Elimination of these subjects from the analysis continued to show no effect at delay 1. During the second delay, the benefits of pasta weakened relative to water and only approached significance $(p=0.068)$, while no effects were seen with bread consumption.

Integrated summary of cognitive domains

Table 2 provides a summary of findings based on each cognitive test. In general, low-GI carbohydrate food intake was associated with better cognitive performance relative to consumption of the high-GI carbohydrate food.

\section{Discussion}

This study highlights the vulnerability to cognitive decrements following meal ingestion and the ability of different types of carbohydrate foods to modulate cognitive performance during the postprandial period in adults with type 2 diabetes. While not all cognitive domains assessed were influenced by carbohydrate intake, for those that were, performance was generally superior following ingestion of the low-GI compared with the high-GI carbohydrate meal. Indeed, for delayed verbal recall, a strong negative association between postprandial gAUC and memory performance was apparent, with both the GI of the carbohydrate meals and individual differences in response to their ingestion contributing to the variance in gAUC and consequent memory recall.

Increasingly evident is that transient periods of hyperglycaemia may be detrimental to cognitive performance in

Table 2 Overview of findings by cognitive task and analyses

\begin{tabular}{|c|c|c|c|}
\hline Cognitive task & \multicolumn{3}{|l|}{ Treatments } \\
\hline \multicolumn{4}{|l|}{ Word list } \\
\hline Global analysis & $\mathrm{P}$ is better than $\mathrm{B}$ & NSD & $\mathrm{P}$ is better than $\mathrm{W}$ \\
\hline Immediate recall $^{\mathrm{a}}$ & NSD & NSD & NSD \\
\hline Delayed recall & $\mathrm{P}$ is better than $\mathrm{B}$ & $\mathrm{B}$ is worse than $\mathrm{W}$ & $\mathrm{P}$ is better than $\mathrm{W}$ \\
\hline Global analysis & $\mathrm{P}$ is better than $\mathrm{B}$ & NSD & $\mathrm{P}$ is better than $\mathrm{W}$ \\
\hline Immediate recall $^{\mathrm{b}}$ & NSD & NSD & NSD \\
\hline Delayed recall & $\mathrm{P}$ is better than $\mathrm{B}$ & NSD & $\mathrm{P}$ is better than $\mathrm{W}$ \\
\hline Time of food effect & First and second delay & First and second delay & Second delay \\
\hline \multicolumn{4}{|l|}{ Verbal-paired associates } \\
\hline Time of food effect & First and second delay & First and second delay & First and second delay \\
\hline \multicolumn{4}{|l|}{ Digit-span forward } \\
\hline Global analysis & NSD & NSD & NSD \\
\hline Digit recall & $\mathrm{P}$ is better than $\mathrm{B}$ & NSD & $\mathrm{P}$ is better than $\mathrm{W}$ \\
\hline Time of food effect & First delay & First and second delay & First delay \\
\hline \multicolumn{4}{|l|}{ Trail making } \\
\hline Global analysis (part a) ${ }^{\mathrm{c}}$ & NSD & NSD & NSD \\
\hline Global analysis (part b) & $\mathrm{P}$ is better than $\mathrm{B}$ & NSD & NSD \\
\hline \multicolumn{4}{|l|}{ Test of everyday attention } \\
\hline \multicolumn{4}{|l|}{ Without distraction } \\
\hline Global analysis & NSD & $\mathrm{B}$ is better than $\mathrm{W}$ & $\mathrm{P}$ is better than $\mathrm{W}$ \\
\hline
\end{tabular}

$P$ pasta, $B$ bread, $W$ water, $N S D$ not significantly different

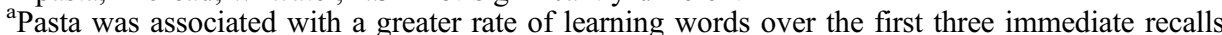

${ }^{b}$ No significant difference observed with immediate recall in both paragraphs

${ }^{c}$ Difference between first and second administrations 
adults with type 2 diabetes [4-6], with this study demonstrating that meal-induced changes in blood glucose are of sufficient magnitude to affect performance adversely. Admittedly, performance following pasta or bread consumption, compared with performance on water days, did not provide a consistent pattern of benefit following pasta consumption and decrement following bread intake. By contrast, comparisons between bread and pasta treatments, which allowed the control of energy intake and stimulation of gut signals associated with meal ingestion, provided a consistent pattern implicating the greater hyperglycaemic response following bread consumption as a fundamental mediator of the cognitive responses observed. Specifically, in measures where sensitivity to food consumption was observed, including delayed verbal memory, working memory, executive function and auditory selective attention, performance following ingestion of the low-GI meal was superior to that following the high-GI meal. Nevertheless, some cognitive functions, including sustained attention, showed little or no sensitivity to the type of carbohydrate food consumed. While benefits associated with pasta consumption, relative to bread, were not apparent on delayed VPA recall, as they were for word list and paragraph recall, response following the low-GI carbohydrate intake trended in a positive direction during VPA assessment. The degree to which differences in test versions of the VPA, while adjusted for in the analyses, confounded these results cannot be determined. Importantly, carbohydrate ingestion, irrespective of type of carbohydrate consumed, improved measures of sustained attention, suggesting that differences in response to bread and pasta consumption cannot be explained by more generalised differences in arousal levels following meal ingestion.

Comparable cognitive performance decrements, including executive function, but a lack of change in other cognitive domains such as sustained attention and immediate verbal recall were observed in this study following the high- vs low-GI carbohydrate meal compared with the decrements reported by Sommerfield and colleagues, where similar levels of blood glucose were attained using a hyperglycaemic/hyperinsulinaemic clamp [5]. Collectively, these results support an argument that more robust hyperglycaemia-induced decrements are apparent in more complex tasks [5]. Whether this simply reflects ceiling effects on less demanding tasks and/or a lack of sensitivity of these tasks in capturing modest performance decrements cannot be ascertained by this study. Nevertheless, poorer performance is observed in several tasks relying on different brain regions for successful completion. Thus, the acute elevations in blood glucose produced by the present study meals or the implementation of a hyperglycaemic/ hyperinsulinaemic clamp [5] result in reasonably diffuse changes in cognitive performance, with little evidence that one specific cognitive domain and/or brain region is spared over another.

Evidence from chronic studies in animal models [16] and human trials $[1,2]$ point to extreme susceptibility of the hippocampus and related structures to the adverse effects of diabetes. Indeed, preliminary analyses of the current data, to determine predictors of task performance under the water condition, revealed that higher $\mathrm{HbA}_{1 \mathrm{c}}$ levels associated with poorer delayed memory recall (assessed as loss of information over the delay period) on paragraph recall for the first story $\left(R^{2}=0.39, p=0.0046\right.$, for the first delay and $R^{2}=0.54, p<0.0001$, for the second delay), supporting the argument that poorer metabolic control is associated with greater cognitive dysfunction, at least in older adults with type 2 diabetes $[1,2,4]$. Results from this and our previous work [4] show that performance in tasks, including delayed verbal memory, which are dependent on hippocampal function can be further suppressed in the face of meal-induced hyperglycaemia. While our results are consistent with enhanced vulnerability of the hippocampus and related structures to type 2 diabetes, no impact of hyperglycaemia was observed on delayed verbal memory performance in subjects undergoing a hyperglycaemic/ hyperinsulinaemic clamp [5]. Two factors may contribute to this discrepancy. The first is a potential role of insulin in the decrements observed [17]. That is, comparisons between hyperglycaemia and euglycaemia in the clamp study were both done under hyperinsulinaemic conditions, while comparisons in the present study were relative to fasting (water) conditions, where presumably insulin levels were substantively lower. The second potential contributor to the differences in results obtained relates to the fact that the second delay period in the present study, where the more robust effect of meal consumption was observed, was probably longer than that used by Sommerfield et al. [5], pointing to potential differences in task complexity.

At present, there is no known metabolic mechanism linking acute postprandial hyperglycaemia to the cognitive dysfunction observed. Indeed, the present study cannot distinguish between a primary effect of hyperglycaemia per se and other metabolic and hormonal changes that occur concurrently, including hyperinsulinaemia. Although numerous mechanisms relating to chronic hyperglycaemia and hyperinsulinaemia have been proposed to explain the overall lower cognitive performance and increased dementia risk in adults with type 2 diabetes [2, 18], these mechanisms are unlikely to be operative within the time frame of a postingestive response. Studies relating to changes in brain glucose transporter (GLUT) mRNA levels in streptozotocin-induced diabetic rats [19] and reduced translocation of hippocampal GLUT4 transporters to the plasma membrane in hippocampal neurons in streptozotocin-induced [19] and obese Zucker rats [16] point to potential changes in neuronal glucose uptake and intracellular utilisation, which may be of relevance to the 2-h postprandial period examined in the present study. Similarly, euglycaemic/hyperinsulinaemic clamp studies in animals, healthy seniors and those with Alzheimer disease, reporting enhancements in hippocampal-dependent cognitive function, demonstrate the sensitivity of hippocampal neurons to transitory changes in local insulin concentrations [20]. This mechanism may be of relevance to postingestive responses, assuming that blood insulin levels would parallel levels observed with glucose. Thus, other 
metabolic and/or endocrine factors, beyond the reported association between delayed verbal memory recall and postprandial gAUC, require consideration.

In summary, food-induced hyperglycaemia related to the GI of the carbohydrate consumed and individual variability in response to carbohydrate ingestion, was found to be associated with cognitive function in adults with type 2 diabetes. Specifically, subjects who showed the greatest postprandial rise in blood glucose levels were the ones who displayed the poorest memory performance after meal ingestion. These findings suggest that adults with type 2 diabetes are susceptible to poorer cognitive performance, especially in more complex tasks, following ingestion of meals that result in large postprandial increases in blood glucose. This points to the need to adopt diet strategies to minimise these excursions in blood glucose levels.

Acknowledgements We would like to thank V. Vuksan and A. Jenkins for their assistance with subject recruitment and use of their study facility. The Natural Sciences and Engineering Research Council of Canada supported this research (grant no. 2769-01). There was no conflict of interest associated with the completion of this research.

\section{References}

1. Awad N, Gagnon M, Messier C (2004) The relationship between impaired glucose tolerance, type 2 diabetes, and cognitive function. J Clin Exp Neuropsychol 26:1044-1080

2. Strachan MW, Deary IJ, Ewing FM, Frier BM (1997) Is type II diabetes associated with an increased risk of cognitive dysfunction? A critical review of published studies. Diabetes Care 20:438-445

3. Cox D, Gonder-Frederick L, McCall A, Kovatchev B, Clarke W (2002) The effects of glucose fluctuation on cognitive function and QOL: the functional costs of hypoglycaemia and hyperglycaemia among adults with type 1 or type 2 diabetes. Int J Clin Pract 129:20-26

4. Greenwood CE, Kaplan RJ, Hebblewaithe S, Jenkins DJ (2003) Carbohydrate induced memory impairment in adults with type 2 diabetes mellitus. Diabetes Care 26:1961-1966
5. Sommerfield AJ, Deary IJ, Frier BM (2004) Acute hyperglycaemia alters mood state and impairs cognitive performance in people with type 2 diabetes. Diabetes Care 27:2335-2340

6. Cox DJ, Kovatchev BP, Gonder-Frederick LA et al (2005) Relationships between hyperglycaemia and cognitive performance among adults with type 1 and type 2 diabetes. Diabetes Care 28:71-77

7. Beck AT, Ward CH, Mendelson M, Mock J, Erbaugh J (1961) An inventory for measuring depression. Arch Gen Psychiatry 4:561-571

8. Shipley WC (1940) A self-administering scale for measuring intellectual impairment and deterioration. J Psychol 9:371-377

9. Wolever TM, Jenkins DJ, Jenkins AL, Josse RG (1991) The glycemic index: methodology and clinical implications. Am J Clin Nutr 54:846-854

10. Brandt J (1991) The Hopkins verbal learning test: development of a new memory test with six equivalent forms. Clin Neuropsychol 5:125-142

11. Wechsler D (1987) Wechsler memory scale-revised. Psychological Corporation, New York

12. Wechsler D (1997) Wechsler memory scale, 3rd edn. Psychological Corporation, New York

13. Kaplan RJ, Greenwood CE, Winocur G, Wolever TM (2000) Cognitive performance is associated with glucose regulation in healthy elderly persons and can be enhanced with glucose and dietary carbohydrates. Am J Clin Nutr 72:825-836

14. Reitan RM, Wolfson D (1985) The Halstead-Reitan neuropsychological test battery. Neuropsychology Press, Tucson, Arizona

15. Robertson IH, Ward T, Ridgeway V, Nimmo-Smith I (1994) The test of everyday attention (TEA). Neurological tests and assessments for adults. Thames Valley Test Company, Oxford

16. Winocur G, Greenwood CE, Piroli GG et al (2005) Memory impairment in obese Zucker rats: an investigation of cognitive function in an animal model of insulin resistance and obesity. Behav Neurosci 119:1389-1395

17. Craft S, Watson GS (2004) Insulin and neurodegenerative disease: shared and specific mechanisms. Lancet Neurol 3: $169-178$

18. Craft S, Asthana S, Cook DG et al (2003) Insulin dose-response effects on memory and plasma amyloid precursor protein in Alzheimer's disease: interactions with apolipoprotein E genotype. Psychoneuroendocrinology 28:809-822

19. McEwen BS, Reagan LP (2004) Glucose transporter expression in the central nervous system: relationship to synaptic function. Eur J Pharmacol 490:13-24

20. Park CR (2001) Cognitive effects of insulin in the central nervous system. Neurosci Biobehav Rev 25:311-323 